\title{
Oligoclonal bands and levels of interleukin 4 , interleukin 10, and tumor necrosis factor alpha in idiopathic intracranial hypertension Egyptian patients
}

Mohamed S. El-Tamawy', Maha A. Zaki', Laila A. Rashed², Eman H. Esmail', Shaimaa Shaheen Mohamed ${ }^{1}$ and Wesam Osama $^{1 *}$

\begin{abstract}
Background: Idiopathic intracranial hypertension $(\mathrm{IIH})$ is a neurological disorder of unknown pathophysiology with many proposed theories that involve CSF dynamics but recently, involvement of inflammatory and autoimmune processes has been postulated.

Objectives: To investigate presence of oligoclonal bands (OCB) in cerebrospinal fluid (CSF) and serum cytokine level in patients with $\| \mathrm{H}$.

Methods: This study was conducted on $27 \mathrm{lH}$ female patients and 21 age- and sex-matched control groups. Patient and control groups were subjected to measurement of interleukin-4 (IL-4), IL-10, and tumor necrosis factor a (TNF-a) levels in serum, and CSF oligoclonal bands was measured in the IIH patient group. Body mass index (BMI) was measured to both patients and control.

Results: Serum levels of IL-4, IL-10, and TNF alpha were significantly higher in IIH patients than controls $(p<0.001)$; $22 \%$ of IIH patients had positive OCB in CSF. There was a statistically significant difference regarding TNF-a level in OCB-positive and OCB-negative patients being higher in positive patients. No statistically significant correlation was found between serum levels of IL-4, IL-10, TNF- $a$, and BMI.
\end{abstract}

Conclusion: Autoimmune inflammatory process may play a role in pathophysiology of $\mathrm{IH}$.

Keywords: Idiopathic intracranial hypertension, IL-4, IL-10, TNF-a in IIH patients, OCB in IIH

\section{Introduction}

Idiopathic intracranial hypertension (IIH) is a neurological disorder of increased intracranial tension in the absence of a structural lesion or other identifiable reason [1]. The name idiopathic intracranial hypertension assumes that no underlying cause for raised ICP has been identified. IIH diagnosis is made in patients who satisfy the modified Dandy criteria [2].

There are many proposed theories which mostly involve CSF dynamics to explain the etiology of IIH [3]. Recently, involvement of inflammatory and autoimmune mechanisms has been postulated as IIH is commonly

\footnotetext{
* Correspondence: Wesamosama9@yahoo.com

${ }^{1}$ Neurology Department, Faculty of Medicine, Cairo University, Giza, Egypt Full list of author information is available at the end of the article
}

associated with autoimmune diseases such as autoimmune thyroiditis or systemic lupus erythomatosus [4]. Positive oligoclonal bands (OCBs) of immunoglobulin G (IgG) in CSF give an evidence for the occurrence of a humoral immune response [5]. In a previous study, Altıkka-Uzun and colleagues [6] found OCBs in 30.77\% of IIH patients involved in their study. Cytokines are a group of proteins that are involved in regulation of immune and inflammatory systems [7]. In previous studies, it was found that patients with IIH had highly elevated levels of TNF- $a$, IFN-g, IL-4, IL-10, IL-12, and IL-17 in their sera compared with controls $[4,6]$. The aims of this study are to investigate the presence of oligoclonal bands (OCBs) in CSF and serum cytokine levels in 
patients with IIH and their relation to the severity of the condition.

\section{Subjects and methods}

This was a case control study conducted on 27 Egyptian female patients diagnosed as IIH according to modified Dandy criteria [2]. They were collected randomly from neurology department and outpatient clinic in Kasr El Ainy Hospital. The age of patients ranged from 1848 years. Twenty-one normal females matched for age were also included as a control group.

This sample size was used as in a previous study [6]; the difference in IL-4 and IL-10 levels between IIH patients and the healthy controls was $2.5 \mathrm{pg} / \mathrm{ml}$ with pooled standard deviation of $2.4 \mathrm{pg} / \mathrm{ml}$. A minimum sample size of 21 cases in each group is required to elicit this difference at an alpha level of 0.05 and a power of the study of $90 \%$. Sample size was calculated using Gpower software ver. 3.1.9.2.

Patients with other neurological disorder or with a reason that may explain intracranial hypertension as patients on steroids or patients with immunologic disorder as systemic lupus erythematosus (SLE) were excluded.

All subjects in this study were subjected to full history taking including history of hormonal contraception, vitamin A, antibiotic, or any other drug intake, and were subjected also to full neurological examination. Body mass index (BMI) was calculated for patients and control groups. Ophthalmological assessment including perimetry (visual field examination), magnetic resonance imaging (MRI) on brain, and magnetic resonance venography (MRV) were performed on 1.5 Tesla Phillips Intera scanner at the Magnetic Resonance Unit (Radio diagnosis Department, Kasr AL Ainy Hospital) for patients with IIH. Lumber puncture and CSF pressure measurement was done for patients with IIH with assessment for presence of OCB by gel electrophoresis using IgG-IEF kits supplied by Helena Bioscience Europe (England), in Biochemistry Department of Kasr EL-Aini Hospitals, where freshly collected undiluted CSF samples were tested immediately using a PER IEF electrode assembly. Following focusing, it is placed on pre-wet transfer membranes for $10-20 \mathrm{~s}$ each time with developing a weight on it for $30 \mathrm{~min}$ then the transfer membrane is put with its red dot up on a staining dish containing a blocking solution the surface of membrane should be covered and gently agitated for $30 \mathrm{~min}$ then the membrane is washed by purified water then saline solution several times then its left to allow color to develop for 20 min with gentle agitation. Finally, the membrane is washed again and left to dry. The IgG bands become visible as a red or red brown color on the membrane.

Laboratory investigation were done for patients and control groups to measure serum levels of IL-4, IL-10, and TNF- $\alpha$ using ELISA kits supplied by e bioscience (USA/San Diego) in Biochemistry Department of Kasr EL-Aini Hospitals, where an anti-human (IL-4, IL-10, or TNF- $\alpha$ ) coating antibody is adsorbed onto microwells. Human (IL-4, IL-10, or TNF- $\alpha$ ) in the sample binds to antibodies adsorbed to the microwell. Following incubation, the unbound biotin-conjugated anti-human (IL-4, IL-10, or TNF- $\alpha$ ) antibody is removed during wash step. Streptavidin horseradish peroxidase (HRP) is added and binds to the biotin-conjugated anti-human (IL-4, IL-10, or TNF- $\alpha$ ) antibody. A colored product is formed in proportion to the amount of human (IL-4, IL-10, or TNF- $\alpha$ ) present in the sample or standard. The reaction is terminated by addition of acid and absorbance is measured at $450 \mathrm{~nm}$. CSF examination for these cytokine levels and comparison of them between both groups was not conducted in the current studies due to technical difficulties of obtaining CSF samples from the healthy control group.

\section{Statistical method}

Data were coded and entered using the statistical package SPSS (Statistical Package for the Social Sciences) version 24. Data were summarized using mean, standard deviation, median, minimum and maximum in quantitative data, and using frequency (count) and relative frequency (percentage) for categorical data. Comparisons between quantitative variables were done by the nonparametric Kruskal-Wallis and Mann-Whitney tests. For comparing categorical data, chi-square $\left(\chi^{2}\right)$ test was performed. Correlations between quantitative variables were done by Spearman correlation coefficient. $p$ values less than 0.05 were considered statistically significant.

\section{Results}

Age of patients in this study was ranged from 18-40 with a mean age of $28.81 \pm 6.91$ years, and for controls, it ranged from 25 to 38 years with a mean of $30.67 \pm 3.71$ years. There was no statistically significant difference between cases and control in BMI.

Results of this study showed that $37 \%$ of patients had moderate headache, $29.7 \%$ had severe headache, $29.7 \%$ had mild headache, and $3.7 \%$ had no headache. A total of $66.7 \%$ of patients did not suffer from tinnitus while $33.3 \%$ suffered from it. Nausea was present in $77.8 \%$ while $22.2 \%$ did not complain of it, while no patients complained of vomiting. A total of $77.8 \%$ of patients did not complain of phonophobia during their disease course and $22.2 \%$ suffered from it; also, $51.9 \%$ of patients did not suffer from photophobia and $48.1 \%$ patients complained of it. A total of 59.3\% did not complain of TVOs and $40.7 \%$ suffered from it. A total of $33.3 \%$ of patients had mild field defect, $29.6 \%$ had sever field changes, $25.9 \%$ did not suffer from field defects, and 
Table 1 Comparison of patient and control groups regarding levels of cytokines

\begin{tabular}{|c|c|c|c|c|c|c|c|c|c|}
\hline & \multicolumn{4}{|l|}{ Case } & \multicolumn{4}{|c|}{ Control } & \multirow[t]{2}{*}{$p$ value } \\
\hline & Mean & SD & Minimum & Maximum & Mean & SD & Minimum & Maximum & \\
\hline IL4 (pg/ml) & 95.50 & 26.84 & 50.50 & 143.70 & 13.14 & 3.80 & 4.70 & 18.40 & $<0.001$ \\
\hline IL10 (pg/ml) & 84.16 & 27.65 & 41.60 & 117.20 & 22.26 & 22.15 & 2.30 & 80.00 & $<0.001$ \\
\hline TNF- $a(p g / m l)$ & 118.52 & 36.38 & 65.80 & 201.70 & 7.34 & 5.45 & 0.10 & 17.60 & $<0.001$ \\
\hline
\end{tabular}

SD standard deviation, IL4 interleukin 4, IL 10 interleukin 10, TNF- $a$ tumor necrosis factor alpha, $\mathrm{pg} / \mathrm{m} / \mathrm{pictogram} / \mathrm{milliliter}$

$11.1 \%$ had moderate field changes. A total of $65.4 \%$ of patients had normal MRI and MRV, unilateral transverse sinus stenosis was present in $26.9 \%$, and bilateral transverse sinus stenosis was present in $7.7 \%$. Oligoclonal bands were positive in $22.2 \%$ and negative in $77.8 \%$.

In this study, there was a statistically significant difference between patients and control groups regarding IL4 , IL-10, TNF- $\alpha(p<0.0001)$ as illustrated in Table 1 . A statistically significant difference $(p=0.033)$ was found between $\mathrm{OCB}+\mathrm{ve}$ patients and $\mathrm{OCB}$-ve patients regarding duration of illness being higher in $\mathrm{OCB}+\mathrm{ve}$ patients. Statistically significant difference was found between levels of TNF- $\alpha$ in OCB +ve and -ve patients $(p<0.001)$ as illustrated in Table 2. Also, there was a significant difference in BMI in patients with or without history of TVOs $(p \leq 0.001)$, tinnitus $(p=0.031)$, and visual field deterioration $(p=0.009)$.

There was a strong statistically significant negative correlation between serum level of IL-10 and age of patients and $(p=0.001, r=-0.589)$ and also between it and age of onset of IIH $(p=0.001, r=-0.622)$ while no statistically significant correlation was found between BMI and levels of IL-4, IL-10, and TNF- $\alpha$ ( $p \geq 0.05)$, as shown in Table 3 . There was a statistically significant positive correlation between serum level of TNF- $\alpha$ and opening pressure $(p=0.047, r=0.386)$ as shown in Table 4. Also, there was a statistically significant positive relation between BMI and opening pressure $(p=0.002$, $r=0.571)$.

\section{Discussion}

Involvement of inflammatory mechanisms and changes in cytokine levels has been proposed recently as a theory for IIH pathophysiology especially as it is sometimes associated with immunologic disorders such as systemic lupus erythematosus (SLE) and antiphospholipid syndrome and it may also show a fluctuating course with "remission and relapses" like that seen in autoimmune disorders. However, the exact pathophysiology of it is still not clear $[8,9]$.

Interleukin-4, IL-10, and TNF- $\alpha$ are involved in the pathogenesis of autoimmune disorders such as SLE [10] which is commonly associated with IIH. There is growing evidence showing that cytokines play crucial roles in the pathology of various neurological disorders and therefore are potential targets for the development of novel and effective therapeutics for central nervous system diseases [11]. Accordingly, guided by the theory of involvement inflammatory mechanisms in IIH pathogenesis, this study aimed to detect the serum levels of IL-4, IL-10, and TNF- $\alpha$ and the presence of OCB in CSF in IIH patients and their relation to the severity of the condition.

In this study, a statistical highly significant difference was found between IIH patients group $(n=27)$ and control group $(n=21)$ as regards to the levels of IL-4, IL-10, and TNF- $\alpha$ being higher in IIH patients than controls $(p<0.001)$; our results agreed with Altıokka-Uzun and colleagues [6] and with Dhungana and colleagues [12]. While it disagreed with Edwards and colleagues [4] who found no significant difference in serum levels of these cytokines between IIH patients and healthy controls while they were higher in the CSF of IIH patients than healthy controls. It may be explained by intrathecal synthesis of these cytokines that may diffuse to the serum [4].

Samanci and colleagues [13] disagreed with our results as they found that levels of TNF- $\alpha$ were significantly lower in IIH patients than in normal controls $(p=0.008)$; this difference can be explained by using different methods in detection of TNF- $\alpha$, as they used multiplex immunoassay while this study used the ELISA method, also their study included IIH patients with other comorbidities including

Table 2 Comparison of levels of IL-4, IL-10, and TNF-a in OCB +ve and -ve patients

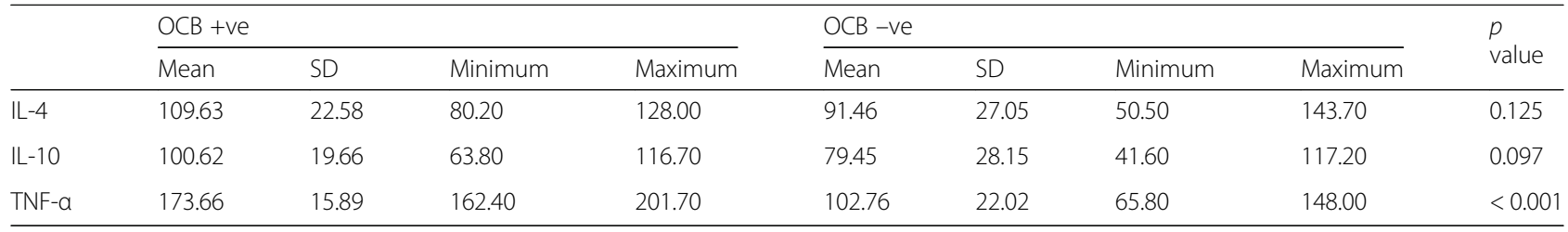

OCB oligoclonal band, +ve positive, -ve negative, SD standard deviation, IL4 interleukin 4, IL10 interleukin 10, TNF- $a$ tumor necrosis factor alpha 
Table 3 Correlations of cytokines levels to age, age of onset, and $\mathrm{BMI}$

\begin{tabular}{lllll}
\hline & & $\mathrm{IL}-4$ & $\mathrm{IL}-10$ & TNF-a \\
\hline Age & Correlation coefficient & -0.148 & -0.589 & -0.165 \\
& $p$ value & 0.462 & 0.001 & 0.410 \\
& $N$ & 27 & 27 & 27 \\
Age of onset & Correlation coefficient & -0.129 & -0.622 & -0.196 \\
& $p$ value & 0.520 & 0.001 & 0.327 \\
& $N$ & 27 & 27 & 27
\end{tabular}

IL4 interleukin 4, IL10 interleukin 10, TNF- $a$ tumor necrosis factor alpha, $N$ number of patients

hypertension, hypothyroidism, diabetes mellitus, psoriasis, and juvenile myoclonic epilepsy while our IIH patients had no comorbidities.

In the present study, it was found that 6 patients out of $27 \mathrm{IIH}$ patients had positive CSF oligoclonal bands which conformed to the results of Samanci and colleagues [13] and Altiokka-Uzun and colleagues [6]. In a study conducted by McLean and colleagues [14] on isoelectric focusing of CSF in patients with different neurological disorders including IIH patients, they found that CSF of IIH patients was negative for local synthesis of IgG by oligoclonal banding.

Results of the current study showed that there was a statistically significant difference between OCB +ve and -ve patients as regards to the duration of illness $(p=0.033)$ being longer in OCB +ve patients with a mean of $(27.5+15.92$ months $)$ than in OCB -ve with a mean of $(11.76+11.25)$ while there were no statistically significant difference between both groups regarding age $(p=0.887)$ or age of onset $(p=0.842)$. These results agreed with Haertle and colleagues [15].

In this study, TNF- $\alpha$ level was significantly higher in $\mathrm{OCB}+$ ve than in OCB -ve patients $(p<0.001)$ while there was no significant difference between both groups as regards to the levels of IL-4 and IL-10 which agreed with Altıkka-Uzun and colleagues [6]. Samanci and colleagues [13] found that there was no significant difference between OCB +ve and OCB -ve IIH patients regarding TNF- $\alpha$ serum level but they did not mention demographics (age, duration of illness, and BMI) or compare comorbidities of both subgroups.

Table 4 Correlations of levels of cytokines to CSF opening pressure in $\mathrm{IH}$ patients

\begin{tabular}{lllll}
\hline & & $\mathrm{IL}-4$ & $\mathrm{IL}-10$ & TNF- \\
\hline $\mathrm{OP} \quad$ Correlation coefficient & 0.353 & 0.097 & 0.379 \\
& $p$ value & 0.071 & 0.632 & 0.05 \\
$N$ & 27 & 27 & 27 \\
\hline
\end{tabular}

OP opening pressure, IL4 interleukin 4, IL 10 interleukin 10, TNF- $a$ tumor necrosis factor alpha, $N$ number
In the current study, it was found that there is statistically significant positive correlation between opening pressure and serum level of TNF- $\alpha$ ( $p=0.047, r=$ 0.386 ) which confirms the inflammatory hypothesis in $\mathrm{IIH}$, and insignificant positive correlation between it and IL-4 $(p=0.071, r=0.353)$ or IL-10 $(p=0.632, r=$ $0.097)$. TNF- $\alpha$ by its proinflammatory role may be related to severity of IIH [6] while it did not conform to Edwards and colleagues [4], this difference can be explained by statistical methods used in each study as they used simple linear regression while we used Pearson's correlation test.

In this study, a statistically significant correlation between BMI and opening pressure $(p=0.002)$ and significant difference in BMI in patients with or without history of TVOs $(p \leq 0.001)$, tinnitus ( $p=$ $0.031)$, phonophobia $(P=0.01)$, and visual field deterioration $(p=0.009)$ were found. This result agreed with Rowe and Sarkies [16] and Bruce and colleagues [15]. This relation to obesity can be explained by that increased intra-abdominal pressure produces raised intracranial pressure by elevating the diaphragm and increasing the intrathoracic pressure, leading to reduced cerebral venous return by the jugular system. This in turn would obstruct cerebral venous outflow, raise cerebral blood volume, and increase intracranial pressure by preventing normal cerebrospinal fluid absorption [16].

This study showed no statistically significant correlation between BMI and levels of IL-4, IL-10, and TNF- $\alpha$ $(p \geq 0.05)$; this result conformed with Altıokka-Uzun and colleagues [6] and Charles and colleagues [17] while it disagreed with Esposito and colleagues [18] who found significant negative correlation between level of IL-10 and BMI.

\section{Conclusion}

The results of this study suggest involvement of an inflammatory process in the pathogenesis of IIH. If confirmed on large sample size and different ethnic groups of IIH patients, these findings would have an impact on the management of the autoimmune inflammatory component of the disease.

\section{Abbreviations}

BMI: Body mass index; ELISA: Enzyme-linked immunosorbent assay;

IIH: Idiopathic intracranial hypertension; IL-10: Interleukin 10; IL-4: Interleukin 4; MRI: Magnetic resonance imaging; OCB: Oligoclonal band; TNF-a: Tumor necrosis factor alpha

\section{Acknowledgements}

The authors acknowledge subjects and Dr Ghada Hatem Abd El Aziz for their participation and cooperation in this study.

\section{Authors' contributions}

MS El-Tamawy is responsible for research idea, data acquisition, data analysis and interpretation, and manuscript writing and reviewing; MA Zaki 
responsible for data acquisition, data analysis and interpretation, and manuscript reviewing; EH Esmail responsible for manuscript writing and reviewing; LA Rashed responsible for interpretation of laboratory data; W Osama responsible for data acquisition, data analysis and interpretation, and manuscript editing; SS Mohamed responsible for manuscript writing. All authors read and approved the final manuscript.

\section{Funding}

This research received no specific grant from any funding agency in the public, commercial, or not-for-profit sectors.

\section{Availability of data and materials}

The datasets generated and/or analyzed during the current study are not publicly available due to current Cairo University regulations \& Egyptian legislation but are available from the corresponding author on reasonable request and after institutional approval.

\section{Ethics approval and consent to participate}

The aim and procedures of the study were explained to every participant and all participants provided informed written consent to participate in the study. The study was approved by the ethical committee of department of Neurology, Faculty of medicine, Cairo University, on October 20, 2014, but Cairo University does not provide approval reference number.

\section{Consent for publication}

Not applicable.

\section{Competing interests}

The authors declare that the research was conducted in absence of any commercial relationships that could be constructed as a potential competing interest.

\section{Author details}

${ }^{1}$ Neurology Department, Faculty of Medicine, Cairo University, Giza, Egypt.

${ }^{2}$ Biochemistry Department, Faculty of Medicine, Cairo University, Giza, Egypt.

Received: 5 April 2019 Accepted: 29 November 2019

Published online: 19 December 2019

\section{References}

1. Friedman D, Liu G, Digre K. Revised diagnostic criteria for the pseudotumor cerebri syndrome in adults and children. Neurology. 2013;81:1159-65.

2. Friedman DI, Jacobson DM. Diagnostic criteria for idiopathic intracranial hypertension. Neurology. 2002;59(10):1492-5.

3. Wakerley B, Tan M, Ting E. Idiopathic intracranial hypertension. Cephalalgia. 2015;35:248-61.

4. Edwards LJ, Sharrack B, Ismail A, Tench CR, Gran B, Dhungana S, et al. Increased levels of interleukins 2 and 17 in the cerebrospinal fluid of patients with idiopathic intracranial hypertension. Am J ClinExp Immunol. 2013;2:234-44

5. Zeman A, McLean B, Keir G, Luxton R, Sharief M, Thompson E. The significance of serum oligoclonal bands in neurological diseases. J Neurol Neurosurg Psychiatry. 1993;56(1):32-5.

6. Altıokka-Uzun $\mathrm{G}$, Tüzün E, Ekizoğlu E, Ulusoy $C$, Yentür $\mathrm{S}$, Kürtüncü $\mathrm{M}$, et al. Oligoclonal bands and increased cytokine levels in idiopathic intracranial hypertension. Cephalgia. 2015;35(13):1153-61.

7. Cannon JG. inflammatory cytokines in nonpathological states. News Physiol Sci. 2000:15:298-303.

8. McGeeney BE, Friedmann D. Pseudotumor cerebri pathophysiology Headache. 2014:54:445-58.

9. Sbeiti S, Kayed DM, Majuri H. Pseudotumour cerebri presentation of systemic lupus erythematosus: more than an association. Rheumatology. 2003:42(6):808-9.

10. Minami M. Neuro-glio-vascular interaction in ischemic brains. Yakugaku zasshi : Journal of the Pharmaceutical Society of Japan. 2011;131(4):539-44.

11. Sabry A, Sheashaa H, El-Husseini A, Mahmoud K, Eldahshan K, George S, et al. Proinflammatory cytokines TNF- $a$ and IL-6 in Egyptian patients with SLE: its correlation with disease activity. Cytokine. 2006;35(3-4):148-53.

12. Dhungana S, Sharrack B, Woodroofe N. Cytokines and chemokines in idiopathic intracranial hypertension. Headache. 2009;49(2):282-5.
13. Samancı B, Samancı Y, Tüzün E, Altıokka-Uzun G, Ekizoğlu E, İçöz S, et al. Evidence for potential involvement of proinflammatory adipokines in the pathogenesis of idiopathic intracranial hypertension. Cephalalgia. 2017;37(6):1-7.

14. McLean $B$, Luxton $R$, Thompson $E$. A study of immunoglobulin $G$ in the cerebrospinal fluid of 1007 patients with suspected neurological diseases using isoelectric focusing and the log-lgG index. Brain. 1990;113:1269-89.

15. Haertle M, Kallweit U, Weller M, Linnebank M. The presence of oligoclonal IgG bands in human CSF during the course of neurological diseases. J Neurol. 2014:261(3):554-60.

16. Rowe FJ, Sarkies NJ. The relationship between obesity and idiopathic intracranial hypertension. Int J Obes Relat Metab Disord. 1999;23(1):54-9.

17. Charles BA, Doumatey A, Huang H, Zhou J, Chen G, Shriner D, et al. The roles of IL-6, IL-10, and IL-1RA in obesity and insulin resistance in AfricanAmericans. J Clin Endocrinol Metab. 2011;96(12):E2018-22.

18. Esposito K, Pontillo A, Giugliano F, Giugliano G, Marfella R, Nicoletti G, et al. Association of low interleukin-10 levels with the metabolic syndrome in obese women. J Clin Endocrinol Metab. 2003:88(3):1055-8.

\section{Publisher's Note}

Springer Nature remains neutral with regard to jurisdictional claims in published maps and institutional affiliations.

\section{Submit your manuscript to a SpringerOpen ${ }^{\circ}$ journal and benefit from:}

- Convenient online submission

- Rigorous peer review

- Open access: articles freely available online

- High visibility within the field

- Retaining the copyright to your article

Submit your next manuscript at $\boldsymbol{\nabla}$ springeropen.com 